\title{
Acute Eosinophilic Pneumonia Following Cigarette Smoking
}

\author{
Yutaro Shiota, Tohru Kawai*, Hitomi Matsumoto, Junichiro Hiyama*, Yoshiyuki Tokuda*, \\ Masaomi MaruKawa*, Tetsuya ONO* and Hiroto MashibA*
}

\begin{abstract}
Two cases of acute eosinophilic pneumonia following cigarette smoking are analyzed for characteristic features. The first patient noted dyspnea 14 days after initiation of smoking. The second patient noted dyspnea 12 days after beginning to smoke. Both cases had characteristic features including occurrence at an age younger than 30 years; less than 1 month duration of cigarette smoking before onset of disease; and no identifiable cause of acute eosinophilic pneumonia apart from smoking. We believe that acute eosinophilic pneumonia following cigarette smoking, which has characteristic features as described above, should be considered as a distinct subtype of AEP.

(Internal Medicine 39: 830-833, 2000)
\end{abstract}

Key words: bronchoalveolar lavage, pleural effusion, glucocorticoid therapy

\section{Introduction}

Allen et al described four cases of acute eosinophilic pneumonia (AEP), an acute febrile illness lasting less than 7 days showing hypoxemia, diffuse pulmonary infiltrates, increased numbers of eosinophils in bronchoalveolar lavage (BAL) fluid, absence of infection or previous atopic illness and a prompt response to corticosteroid therapy (1). Based on our review of two cases of AEP following cigarette smoking, we believe that AEP associated with cigarette smoking has characteristic features that make it a distinct subtype of this condition.

For editorial comment, see $\mathrm{p} 759$.

\section{Case Report}

\section{Case 1}

A 24-year-old man was admitted to Kure Kyosai Hospital because of fever, cough, and progressive shortness of breath.
The patient had been well until 5 days earlier when he noted fever, nausea, productive cough, and dyspnea. Antibiotic treatment prior to hospitalization did not result in improvement, and dyspnea increased. After a radiograph of the chest revealed pulmonary infiltrates, the patient was referred to our hospital. He had no previous history of asthma or atopic disease. The patient had started to smoke 10 cigarettes per day 18 days before admission but quit smoking 6 days before admission. He recalled previously smoking a cigarette once when he was 13 and again when 22 years old. No medications had been taken prior to this illness. Physical examination revealed a young man in mild respiratory distress. His temperature was $38.3^{\circ} \mathrm{C}$, and his chest was clear to auscultation. Cardiac examination was normal. The initial chest roentgenogram showed bilateral ground-glass opacities and a left-sided pleural effusion (Fig. 1A). Computed tomography of the chest revealed a patchy bilateral distribution of ground-glass opacities. Thickening of interlobular septa and bronchovascular bundles, was seen, as well as a left pleural effusion (Fig. 1B). Laboratory data on admission included a peripheral white blood cell count of 5,790/ $\mathrm{mm}^{3}$ (differential cell count, $69.3 \%$ neutrophils, $13.7 \%$ lymphocytes, $5.1 \%$ monocytes, $8.9 \%$ eosinophils, and $0.4 \%$ basophils). The serum C-reactive protein concentration was $9.6 \mathrm{mg} /$ dl. Serum immunoglobulin concentrations were: immunoglobulin G, 1,090 mg/ml; immunoglobulin A, $275 \mathrm{mg} / \mathrm{dl}$; immunoglobulin M, $112 \mathrm{mg} / \mathrm{dl}$; and immunoglobulin E, $77 \mathrm{U} /$ $\mathrm{ml}$. Radioallergosorbent testing detected no specific sensitization to allergens. Other venous blood examination results were normal. Arterial blood gas analysis disclosed a pH of 7.41, a $\mathrm{PaCO}_{2}$ of $39.4 \mathrm{mmHg}$, and $\mathrm{a} \mathrm{PaO}_{2}$ of $65.6 \mathrm{mmHg}$ while breathing room air. Sputum cultures were unremarkable. Prednisolone $(30 \mathrm{mg})$ was administered on the second hospital day, after which the patient improved as dyspnea and fever disappeared. On the fifth hospital day, fiberoptic bronchoscopy was performed. Bronchoalveolar lavage (BAL) of the right middle lobe yielded slightly cloudy fluid in which the differential cell count was $6.3 \%$ macrophages, $21.9 \%$ lymphocytes, $1.6 \%$ neutrophils, $1.6 \%$ basophils, and $68.8 \%$ eosinophils. Immunophenotypic analysis of BAL fluid lymphocytes revealed that $76 \%$ of the lymphocytes were $\mathrm{CD} 3+, 17 \%$ were $\mathrm{CD} 4+$, and $63 \%$ were $\mathrm{CD} 8+$. The peripheral blood eosinophil

From the Department of Clinical Research and *the Department of Internal Medicine, Kure Kyosai Hospital, Kure

Received for publication September 10, 1999; Accepted for publication March 10, 2000

Reprint requests should be addressed to Dr. Yutaro Shiota, the Department of Clinical Research, Kure Kyosai Hospital, 2-3-28 Nishichuo, Kure 737-8511 


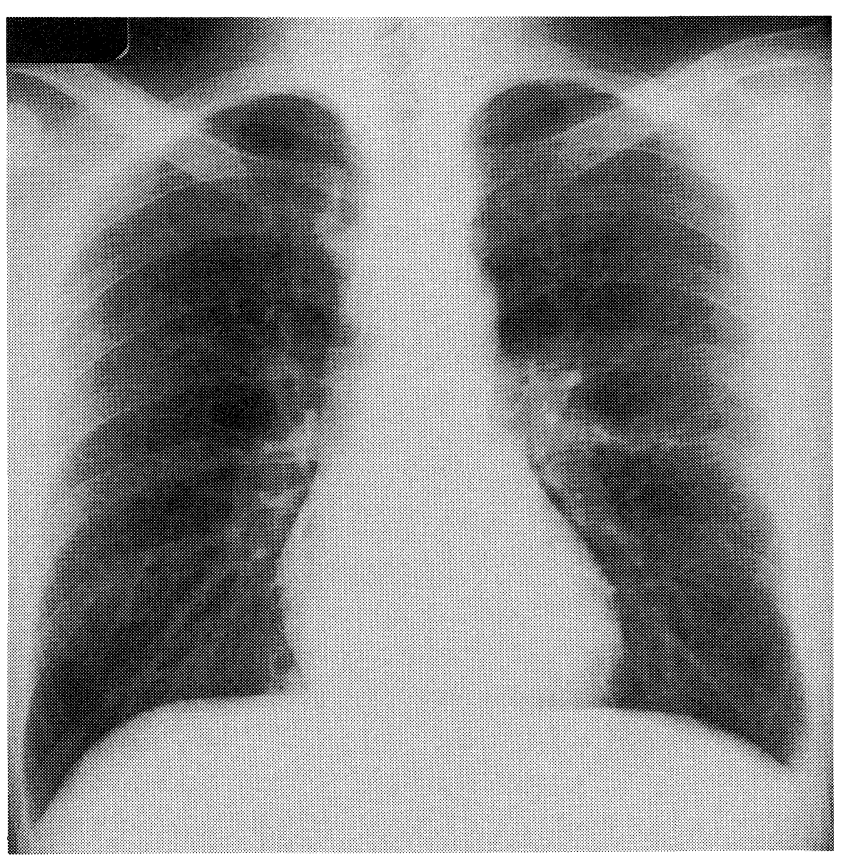

A

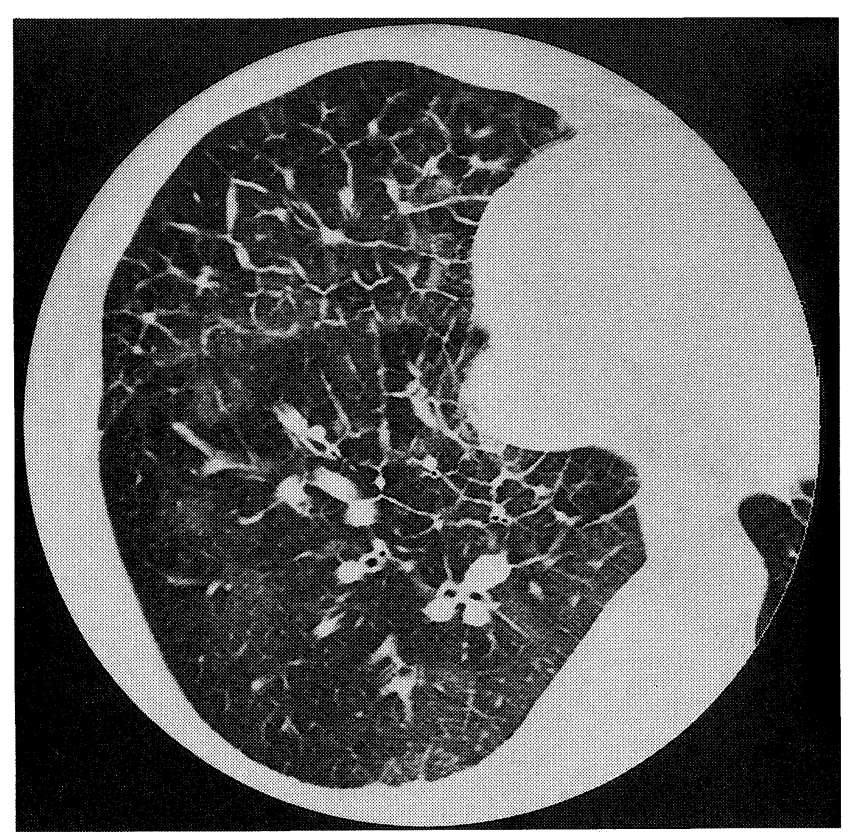

B

Figure 1. Radiologic findings in case 1. A, chest roentgenogram on admission showing bilateral ground-glass opacities and a left pleural effusion. B, computed tomogram of the chest showing patchy distribution of ground-glass opacities. Thickening of interlobular septa and bronchovascular bundles is seen as well as a pleural effusion. count, $8.9 \%$ on admission, increased gradually to $33 \%$ on the eighth hospital day. It subsequently decreased to $5.8 \%$ after discharge. The serum C-reactive protein concentration, evaluated on admission, decreased gradually and was within the normal range on the eighth hospital day. A chest roentgenogram showed complete resolution of abnormalities, and the patient was discharged on the 11th hospital day. One month after discharge, the patient resumed smoking cigarettes for one day; 1 day later he again noted dyspnea and cough, which lasted for 3 days. He then quit smoking and did not resume. Four months later, he continued to be asymptomatic.

\section{Case 2}

A 21-year-old serviceman from the Japanese Self-Defense Forces was admitted to the hospital because of dyspnea and fever. The patient had been well until 1 day before seeking medical evaluation when he noted generalised fatigue, nausea, dyspnea and chills. He saw a physician who diagnosed a "common cold" and initiated treatment with broad-spectum antibiotics and an antipyretic. When the patient's condition deteriorated he was referred to our hospital. He had no previous history of asthma or atopic disease. He had smoked cigarettes once when he was 18 years old and then quit. He began smoking one pack of cigarettes daily 13 days before admission and quit smoking 2 days before admission. On examination the patient was a young man in respiratory distress. His temperature was $39.5^{\circ} \mathrm{C}$. Breath sounds were slightly diminished at both lung bases. Results of a neurologic examination were normal. Radiographs of the chest revealed bilateral diffuse ground-glass opacities and bilateral pleural effusions (Fig. 2A). Noncontrast computed tomography of the chest showed diffuse ground-glass opacification, thickening of interlobular septa and bronchovascular bundles, and bilateral pleural effusions (Fig. 2B). Laboratory data on admission included a peripheral white cell count of $31,300 / \mathrm{mm}^{3}$ (differential cell count, $96 \%$ neutrophils, $2 \%$ lymphocytes, $2 \%$ monocytes and no eosinophils). The serum concentration of C-reactive protein was 15 $\mathrm{mg} / \mathrm{dl}$ and that of immunoglobulin $\mathrm{E}$ was $262 \mathrm{U} / \mathrm{ml}$. Other blood chemistry results were normal. Arterial blood gas analysis revealed a $\mathrm{pH}$ of 7.41, a $\mathrm{PaCO}_{2}$ of $36.1 \mathrm{mmHg}$, and a $\mathrm{PaO}_{2}$ of $51.9 \mathrm{~mm} \mathrm{Hg}$ while breathing room air. A sputum culture yielded normal flora. On the day of admission, intravenous corticosteroid therapy was started with a daily prednisolone dose of $40 \mathrm{mg}$. The patient improved, showing a decrease in pulmonary infiltrates and pleural effusions. On the third hospital day, pleural fluid obtained by thoracocentesis was clear and yellow-tinged, containing cells that included $14 \%$ macrophages, $1 \%$ lymphocytes, $70 \%$ neutrophils, and $15 \%$ eosinophils. BAL fluid obtained by fiberoptic bronchoscopy contained a cell population with $51.1 \%$ macrophages, $22.3 \%$ lymphocytes, $1.1 \%$ neutrophils, and $25.5 \%$ eosinophils. On the fifth hospital day, the daily dose of prednisolone was reduced to $20 \mathrm{mg}$. The initially elevated serum C-reactive protein concentration fell rapidly to the normal range. The peripheral blood eosinophil count, $0 \%$ representing leukocytes on admission, had increased to $41 \%$ by the 12 th hospital day but fell to $12 \%$ by the 19 th hospital 


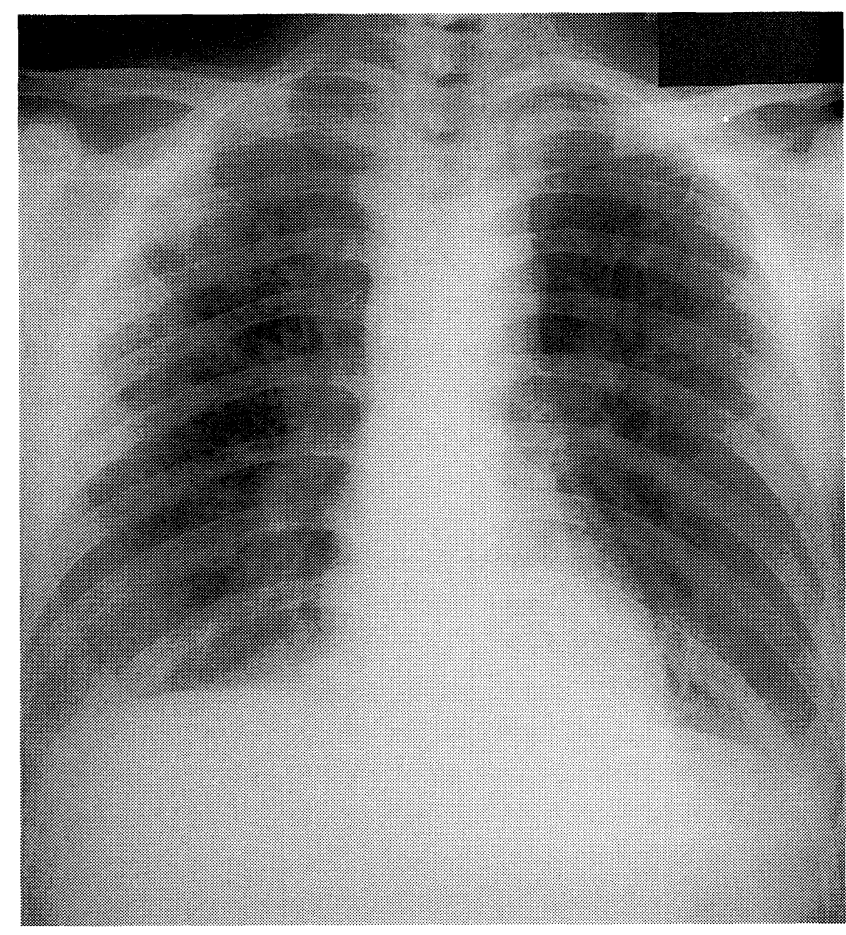

A

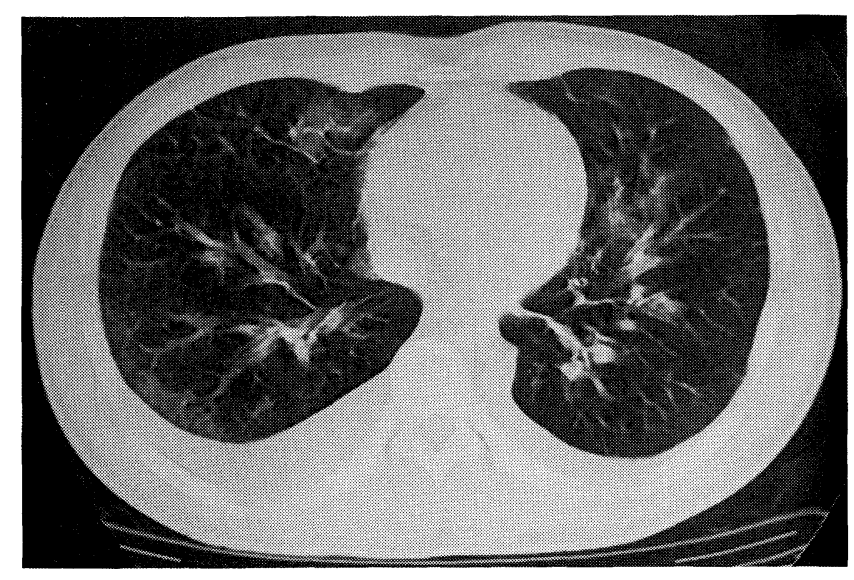

B

Figure 2. Radiologic findings in case 2. A, chest roentgenogram on admission showing bilateral diffuse ground-glass opacities and bilateral pleural effusions. $B$, computed tomogram of the chest showing diffuse ground-glass opacification, thickening of interlobular septa and bronchovascular bundles, and pleural effusions.

day. The patient was discharged in good condition 20 days after admission; at that time chest roentogenography and CT were normal. Three months later, he continued to be asymptomatic.

\section{Discussion}

The present two patients met the criteria for a diagnosis of acute eosinophilic pneumonia by Allen (1): an acute febrile illness of less than 7 days' duration, hypoxemia, diffuse pulmonary infiltrates, an increased percentage of eosinophils in BAL fluid, absence of pulmonary or systemic infections, and complete resolution of pulmonary infiltrates. Hypoxemia was not so severe in case 1 , though. AEP may occur in association with medications (2-7), infections (8-10), inhalation of toxic substances $(11,12)$, or exposure to a variety of biologic agents including fungi (13-16). In the present two cases, we excluded several potential etiologic factors that could induce AEP. In case 1, clarithromycin, ibuprofen, and sulpyrine were administered before admission. In case 2, cefaclor and loxoprofen sodium were administered only once before admission. These drugs are unlikely to have caused AEP since they were administered only after the appearance of such symptoms as fever, cough, and dyspnea. While some infectious microorganisms can cause AEP, no pathogens were detected in any specimens from either patient. Neither patient had a history of exposure to environmental antigens known to cause AEP. It is unlikely that environmental antigens caused AEP in our cases because they remained to be asymptomatic after returning home.

Taki and colleagues have described five patients with AEP who had begun smoking less than 10 days before onset of AEP (17). When four of these patients resumed smoking after the resolution of the disease, only one experienced another episode of AEP. King has reported three patients with AEP who had begun smoking cigarettes within 2 months of onset (18). In reporting a case of AEP associated with cigarette smoking, Nakajima et al stressed the importance of smoking as a cause based on a positive rechallenge test (19). In our case 1, the patient's symptoms upon resuming smoking probably represented another episode of AEP, although no laboratory or roentogenographic confirmation was possible since the patient did not mention the event to a physician until weeks later.

Both patients in this report were younger than 30 years old and had started smoking cigarettes less than 1 month prior to the onset of AEP and had no identifiable cause of AEP apart from smoking. We believe these descriptions define a syndrome of AEP following cigarette smoking. AEP following cigarette smoking should be suspected in young adults when no other etiology for AEP is present. To assess the validity of this diagnosis, we reviewed 61 cases of AEP reported from 1989 through 1998. An adequate history with respect to cigarette smoking was available in 28 cases; 10 patients were nonsmokers and 18 were smokers. Of the 18 occurrences in smokers, 9 met our criteria for AEP following cigarette smoking (Table 1); 8 of these 9 reports originated from Japan (20-25). The ninth case was reported by Godding and colleagues who described AEP in a 14-year-old girl who recently had begun smoking cigarettes, although the authors did not identify smoking as a precipitating cause of AEP in their report (26). We do not know the mechanism by which smoking induces AEP. Nevertheless, 
Table 1. Reported Cases of Acute Eosinophilic Pneumonia Following Cigarette Smoking

\begin{tabular}{clcccc}
\hline Year & Author & Age & Sex & Smoking period & $\begin{array}{c}\text { Number of cigarettes } \\
\text { smoked per day }\end{array}$ \\
\hline 1993 & Hashimoto (20) & 22 & F & 1 month & 10 \\
1995 & Kawaguchi (21) & 18 & F & 1 month & 10 \\
1995 & Nakamura (22) & 21 & M & 1 month & not described \\
1995 & Yamaguchi (23) & 21 & M & 14 days & not described \\
1997 & Kawamura (24) & 19 & M & 3 weeks & 20 \\
1997 & Kawamura (24) & 23 & M & 1 weeks & $20-60$ \\
1997 & Kawamura (24) & 22 & M & 2 weeks & 20 \\
1997 & Sasaki (25) & 18 & M & 2 days & 14 days \\
1998 & Godding (26) & 14 & F & 14 days & not described \\
present cases & & 24 & M & 12 days & 20 \\
\hline
\end{tabular}

we believe that AEP following cigarette smoking is a distinct subtype of AEP that needs to be recognized.

\section{References}

1) Allen JN, Pacht ER, Gadek JE, Davis WB. Acute eosinophilic pneumonia as a reversible cause of noninfectious respiratory failure. $N$ Engl J Med 321: 569-574, 1989.

2) Kondo K, Inoue Y, Hamada H, Yokoyama A, Kohno N, Hiwada K. Acetaminophen-induced eosinophilic pneumonia. Chest 104: 291-292, 1993.

3) Yokoyama A, Mizushima Y, Suzuki H, Arai N, Kitagawa M, Yano S. Acute eosinophilic pneumonia induced by minocycline: Prominent Kerley B lines as a feature of positive re-challenge test. Jpn J Med 29: 195-198, 1990.

4) Shintani H, Minami S, Iwabuchi K, et al. Three cases of minocyclineinduced pneumonitis. Nippon Kyobu Shikkan Gakkai Zasshi (Jpn J Thoracic Dis) 29: 718-723, 1991 (in Japanese, Abstract in English).

5) Horikx PE, Gooszen HC. Minocycline as a cause of acute eosinophilic pneumonia. Ned Tijdschr Geneeskd 136: 530-532, 1992.

6) Bando T, Fujimura M, Noda Y, Hirose J, Ohta G, Matsuda T. Minocyclineinduced pneumonitis with bilateral hilar lymphadenopathy and pleural effusion. Intern Med 33: 177-179, 1994.

7) Ogawa $H$, Kurashima $K$, Namura $M$, et al. Pulmonary infiltrates with eosinophilia due to naproxen. Jpn J Med 30: 32-34, 1991.

8) Mayo J, Collazos J, Martinez E, Diaz F. Acute eosinophilic pneumonia in a patient infected with the human immunodeficiency virus. Tuber Lung Dis 76: 77-79, 1995.

9) Roig J, Romeu J, Riera C, Texido A, Domingo C, Morera J. Acute eosinophilic pneumonia due to toxocariasis with bronchoalveolar lavage findings. Chest 102: 294-296, 1992.

10) Bouchard $O$, Arbib F, Paramelle B, Brambillia C. Acute eosinophilic pneumonia and the larva migrans syndrome: Apropos of a case in an adult. Rev Mal Respir 11: 593-595, 1994.

11) Kelly KJ, Ruffing R. Acute eosinophilic pneumonia following intentional inhalation of Scotchguard. Ann Allergy 71: 358-361, 1993.

12) Brander PE, Tukiainen P. Acute eosinophilic pneumonia in a heroin smoker. Eur Respir J 6: 750-752, 1993.

13) Mouri M, Nambu $Y$, Horii $H$, et al. Case report and review of literature on seasonal distribution and pathogenesis of acute eosinophilic pneumonia in Japan. Nippon Kyobu Shikkan Gakkai Zashhi (Jpn J Thoracic Dis) 31: 1578-1584, 1993 (in Japanese, Abstract in English).
14) Imokawa $S$, Sato A, Taniguchi $M$, et al. Two cases of acute eosinophilic pneumonia with precipitating antibody against Trichosporon cutaneum and Trichoderma viride. Nippon Kyobu Shikkan Gakkai Zasshi (Jpn J Thoracic Dis) 31: 352-359, 1993 (in Japanese, Abstract in English).

15) Godeau B, Brochard L, Theodorou I, et al. A case of acute eosinophilic pneumonia with hypersensitivity to "red spider" allergens. J Allergy Clin Immunol 95: 1056-1058, 1995.

16) Miyazaki E, Sugisaki K, Shigenaga $T$, et al. A case of acute eosinophilic pneumonia caused by inhalation of Trichosporon terrestre. Am J Respir Crit Care Med 151: 541-543, 1995.

17) Taki R, Sawada $M$, Isogai $S$, et al. A possible role of cigarette smoking in the pathogenesis of acute eosinophilic pneumonia. Am J Respir Crit Care Med 153: A271, 1996 (Abstract).

18) King MA, Pope-Harman AL, Allen JN, Christoforidis GA, Christoforidis AJ. Acute Eosinophilic Pneumonia: Radiologic and Clinical Features. Radiology 203: 715-719, 1997.

19) Nakajima M, Manabe T, Niki Y, Matsushima T. Cigarette smoke-induced acute eosinophilic pneumonia. Radiology 207: 829-831, 1998.

20) Hashimoto $S$, Fukabori $T$, Ueda $M$, et al. A case of acute eosinophilic pneumonia. Kokyu (Respiration Research) 12: 502-507, 1993 (in Japanese, Abstract in English).

21) Kawaguchi $H$, Yasuda $H$, Yamashita $K$, et al. A case of acute eosinophilic pneumonia: Bronchoalveolar lavage findings before and after steroid treatment. Nippon Kyobu Shikkan Gakkai Zasshi (Jpn J Thoracic Dis) 33: 738-744, 1995 (in Japanese, Abstract in English).

22) Nakamura $H$, Kashiwabara $K$, Yagyu $H$, et al. Clinical evaluation of three cases of acute eosinophilic pneumonia. Kikanshigaku (J Jpn Soc Bronchol) 17: 432-437, 1995 (in Japanese, Abstract in English).

23) Yamaguchi S, Okubo Y, Hossain M, et al. IL-5 predominant in bronchoalveolar lavage fluid and peripheral blood in a patient with acute eosinophilic pneumonia. Intern Med 34: 65-68, 1995.

24) Kawamura T, Mochizuki Y, Nakahara $Y$, Kimoto T, Watanabe S. A clinical study of 5 cases of acute eosinophilic pneumonia. The relationship between begining to smoke and acute eosinophilic pneumonia. Nippon Kyobu Shikkan Gakkai Zasshi (Jpn J Thoracic Dis) 35: 1252-1258, 1997 (in Japanese, Abstract in English).

25) Sasaki T, Nakajima M, Kawabata S, et al. Acute eosinophilic pneumonia induced by cigarette smoke. Nippon Kyobu Shikkann Gakkai Zasshi (Jpn J Thoracic Dis) 35: 89-94, 1997 (in Japanese, Abstract in English).

26) Godding V, Bodart E, Delos M, et al. Mechanisms of acute eosinophilic inflammation in a case of acute eosinophilic pneumonia in a 14-year-old girl. Clin Exp Allergy 28: 504-509, 1998. 